\title{
I curatores rei publicae nella provincia d'Acaia*
}

\author{
Francesco CAMIA
}

A partire dall'età di Traiano funzionari straordinari di nomina imperiale chiamati curatores rei publicae furono inviati in alcune città dell'Impero a risolvere problemi legati all'amministrazione dei beni municipali. Questi "revisori dei conti», istituiti forse già verso la fine del I sec. d.C. ${ }^{1}$, erano spesso di rango senatorio (od equestre) e generalmente provenivano da una città diversa da quella in cui esercitavano la loro missione. Nel IV sec. d.C. la situazione risulta cambiata : il curator rei publicae si è trasformato in un mero funzionario municipale, scelto tra i membri della locale classe dirigente. Nella parte orientale dell'Impero questi funzionari vengono chiamati $\lambda \mathrm{o} \gamma 1 \sigma \tau \alpha i^{2}$.

Nel presente contributo mi occuperò dei curatores rei publicae attestati nella provincia d'Acaia; ad una rapida rassegna dei logistai, basata sulla documentazione epigrafica, farò seguire alcune considerazioni sulla distribuzione cronologica delle $\mathrm{cu}-$ ratele di città in Acaia e sulla caratterizzazione geografica e sociale dei logistai; infine, mi soffermerò su alcuni aspetti «tecnici». Il presente studio è inevitabilmente condizionato dall'estrema esiguità del campione epigrafico a disposizione, motivo per cui le considerazioni che seguono vogliono

*. Ringrazio C. Berrendonner e M. Cébeillac-Gervasoni, coordinatrici del programma EMIRE, per avermi dato la possibilità di prendere parte al workshop di Parigi, e. M. Christol e J.-L. Ferrary per le preziose osservazioni e i suggerimenti fornitimi in sede di discussione; la mia gratitudine va inoltre a A. D. Rizakis, che mi ha proposto di partecipare all'incontro parigino, e a M. L. Lazzarini per la consueta disponibilità.

1. L. Caesennius Sospes (cos. 114 d.C.) potrebbe aver svolto la funzione di curator coloniarum et municipiorum (in Italia?) nell'età di Domiziano (ILS, 1017; cfr. M. Griffin, Nerva to Hadrian, in The Cambridge Ancient History, 2, XI, Cambridge, 2000, p. 117, nt. 210); contra Camodeca 1980, p. 474-475, è favorevole ad una datazione della curatela di questo perso- essere semplicemente un tentativo di fornire alcuni spunti di riflessione, soggetti al vaglio di nuovi e futuri dati.

\section{LOGISTAI}

Nella provincia d'Acaia sono attestati, tra la fine del II e la fine del IV sec. d.C., sedici curatores rei publicae in quindici città (Atene; Sparta; Patrasso; Trezene; Epidauro; Corone; Asine; Orchomenos d'Arcadia; Tegea; Tebe; Cheronea; Coronea; Amphissa; Hestiea [Eubea]; Andros). Tegea, Cheronea e Patrasso ebbero curatores per due volte, Atene per due o tre volte ${ }^{3}$. Inoltre, la curatela esercitata dal legato imperiale di rango consolare Ti. Claudius Callippianus Italicus (verso il 200 d.C.) riguardò eccezionalmente tutte le città libere dell'Acaia ${ }^{4}$.

T. Flavius Philargyrus ${ }^{5}$, logistes di Orchomenos d'Arcadia nel 192/3 d.C. - quando la città pose una dedica a Settimio Severo $(I G, \mathrm{~V}, 2,346)$ - è il primo curator rei publicae attestato nella provincia d'Acaia; non sembra essere altrimenti noto.

Negli stessi anni (193-195 d.C.), ad Asine, in Messenia, è attestato, in un'altra dedica imperiale (IG, V, 1, 1412), il logistes Flavius Saithidas; quest'ul-

naggio in età traianea (cfr. anche F. Jacques, Les curateurs des cités dans l'Occident Romain de Trajan à Gallien, Parigi, 1983, p. 19-22 e Jacques 1984, p. 3-4 e 7, nr. 1).

2. Mason 1974, p. 66, s.v.; in rari casi, in Asia Minore, sono at-

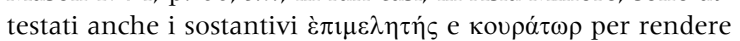
il latino curator rei publicae (Mason 1974, p. 46-47, s.v.; p. 63, s.v.).

3. A seconda che si attribuisca a C. Licinius Telemachus un'unica curatela della durata di quattro anni oppure due curatele separate da un breve intervallo di tempo; vid. infra.

4. Vid. infra. Cfr. $A E, 1980$, nr. 61 (un senatore curator delle città della provincia di Bitinia e Ponto).

5. Roman Peloponnese I, ARC 80. 
timo doveva appartenere ad un ramo della famiglia messenia dei Saithidae che portava il gentilizio Flavius, ed era quindi messenio (se non proprio di Asine) ${ }^{6}$.

In un anno tra il 195 e il 198 d.C. la polis di Hestiea, in Eubea, eresse una statua per Settimio Se-

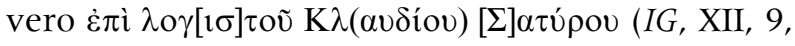
1235). Quest'ultimo sembra essere attestato solo da questa dedica 7 .

Verso la fine del II sec. d.C. un esponente dell'importante famiglia ateniese dei Claudii di Melite ricoprì la funzione di logistes in almeno quattro diverse città (Epidauro, Cheronea, Coronea, Tebe). A causa della lacunosità della dedica eleusinia che lo riguarda $\left(I G, \mathrm{II}-\mathrm{III}^{2}, 3610\right)^{8}$, il personaggio può essere identificato o con Ti. Claudius Sospis "sacerdote dell'altare» o con Ti. Claudius Philippus "daduco ${ }^{9}$. È probabile che egli abbia ricoperto le curatele in successione e non contemporaneamente.

Ad Atene, negli anni iniziali del III sec. d.C. sono attestati due curatores rei publicae, entrambi senatori. Ti. Claudius Subatianus Proculus ${ }^{10}$ è noto da una dedica (in latino) posta dalla città di Cuicul, in Numidia, alle sue figlie (ILS, 9488); l'iscrizione riporta il cursus del personaggio, verosimilmente originario dell'Asia Minore settentrionale ${ }^{11}$. Proculus iniziò la carriera nell'ordine equestre e intorno al 197 d.C. entrò in senato come quaestor urbanus. La sua adlectio, così come il prosieguo della sua carriera senatoria, mostrano il favore di cui godette presso l'imperatore Settimio Severo, di cui fu candidatus al tribunato della plebe (201 d.C.) e alla pretura urbana (204 d.C.). Nel cursus di Subatianus la curatela di Atene e Patrasso è inserita tra la pretura e il comando della legio VI ferrata fidelis constans, databile al più tardi al 207 d.C., dato che ne-

6. Cfr. Roman Peloponnese II, MES 198

7. Cfr. T. E. Gregory, Roman Inscriptions from Aidepsos, in GRBS, 20, 1979, p. 263, nr. 3.

8. SEG, XXV, 213.

9. Cfr. Byrne 2003, p. 160-161, nrr. 164-165 (e p. 538-539).

10. Groag 1939, p. 137-138; Pflaum 1960-1961, nr. 242; H. Devijver, Prosopographia militiarum equestrium quae fuerunt ab Augusto ad Gallienum, I, Lovanio, 1976, C 187 (e Suppl. I, C 187).

11. Cfr. O. Salomies, Die Herkunft des numidischen Legaten Ti. Claudius Subatianus Proculus, in ZPE, 119, 1997, p. 245248; D. Erkelenz, Patria, civis, condecurio - Zur Identifizierung der Herkunft von Rittern und Senatoren in der Römischen Kaiserzeit, in ZPE, 137, 2001, p. 272.

12. Cfr. Jacques 1984, p. 65 s.; Jacques - Scheid 1992, p. 343. gli anni 208-210 egli fu governatore della Numidia. Quella di curator, quindi, sembra essere stata la prima funzione ricoperta da Proculus dopo la pretura, e si daterà nel 205/6 d.C. A questo proposito va rilevato che, almeno in Occidente, la maggior parte dei senatori curatori di città ricoprirono questa funzione dopo la pretura (non necessariamente come prima carica pretoria) ${ }^{12}$. Verso la fine del 210 d.C. Proculus raggiunse il consolato ${ }^{13}$.

C. Licinius Telemachus ${ }^{14}$, originario della LiciaPamphilia, fu curator di Atene nel 209/10 d.C., come attesta un decreto $\left(I G, \mathrm{II}-\mathrm{III}^{2}, 1077=\right.$ Agora, XV, nr. 460) votato dall'Areopago, dal consiglio dei 500 e dall'assemblea popolare a seguito dell'associazione di Geta al potere (sett./ott. 209 o autunno 210) ${ }^{15}$. Il documento riguarda la realizzazione di sacrifici e lo svolgimento di celebrazioni festive, in pubblico e in privato, per tutta la famiglia imperiale; inoltre, decreta la celebrazione del dies imperii di Geta, conformemente agli altri dies imperii, e la distribuzione di donativi al popolo, secondo quanto indicato in un editto (diatagma) dal legatus Augustorum pro praetore e curator della città di Atene C. Licinius Telemachus. L'editto doveva contenere la proclamazione ufficiale dell'avvenuta associazione al potere di Geta e, verosimilmente, anche indicazioni sulla celebrazione del dies imperii. Con ogni probabilità, Licinius Telemachus ricoprì contemporaneamente la funzione di corrector delle città libere dell'Acaia. Il titolo di legatus Augustorum pro praetore, con cui Telemachus viene identificato nel decreto in questione, per quanto in parte integrato, è lo stesso portato da Callippianus Italicus $^{16}$, il quale fu contemporaneamente corrector e curator delle città libere dell'Acaia ${ }^{17}$. Alla carica di corrector, quindi, unica funzione nota della sua carriera pretoria, Licinius Telemachus associò la logisteia di Atene ${ }^{18}$.

13. E. Birley, The Governors of Numidia, A.D. 193-268, in JRS, 40 1950, p. 62, nr. 5 .

14. $P I R^{2}$, L 254; Groag 1939, p. 133-134; Oliver 1973, p. 404; Guerber 1997, p. 218 (nr. 9).

15. D. Kienast, Römische Kaisertabelle : Grundzüge einer römischen Kaiserchronologie ${ }^{2}$, Darmstadt, 1996, p. 166.

16. Vid. infra.

17. Secondo Guerber 1997, p. 218, «la simple mention d'une légation impériale dans une province sénatoriale de la partie hellénophone de l'Empire, lorsqu'elle s'accompagne d'indices qui indiquent qu'un émissaire impérial se charge des affaires d'une cité libre, nous suffit pour l'assimiler à un correcteur".

18. Cfr. Guerber 1997, p. 237-238. 
Il decreto ateniese è il risultato dell'approvazione di una gnome (= proposta) formulata da tre organi : consiglio dell'Areopago, consiglio dei 500, assemblea popolare. Il riferimento alla gnome si spiega in primo luogo in relazione al contenuto del provvedimento. Di norma, infatti, gli onori indirizzati all'imperatore (o alla famiglia imperiale) venivano sottoposti preliminarmente all'approvazione dell'imperatore stesso, il quale poteva accettarli o rifiutarli (o accettarne solo alcuni); è quindi verosimile che gli Ateniesi, prima di approvare in forma definitiva il documento, l'abbiano presentato all'imperatore sotto forma di gno$m e$, perché venisse approvata ${ }^{19}$. In secondo luogo, la presenza di un logistes indica che in quel periodo la polis di Atene non godeva della piena responsabilità finanziaria; le spese relative alla celebrazione del dies imperii di Geta dovevano essere approvate dal logistes. I provvedimenti decisi dalla polis di Atene, quindi, avevano bisogno del via libera dell'imperatore e del curator rei publicae prima di poter essere mandati ad effetto sotto forma di decreto.

Lo stesso personaggio è menzionato in una iscrizione proveniente dal Pireo e datata dall'arconte Dionysios figlio di Dionysios al 212/3 d.C. (IG, II-III'2 , 2963); i membri dell'associazione religiosa dei Paianistai del santuario di Asclepio a Munichia dichiarano di essersi rivolti al logistes di Atene C. Licinius Telemachus su ordine dell'oracolo di Delfi ${ }^{20}$. Due alternative si pongono, entrambe plausibili : o Licinius è stato curator rei publicae di Atene ininterrottamente dal 209/10 al 212/3 d.C., oppure ha

19. Cfr. J. H. Oliver, Greek Inscriptions, in Hesperia, 10, 1941, p. 77-78, nr. 33 : lettera dell'imperatore Adriano relativa ad onori per Antinoo defunto; la parte finale della lettera rivela che si tratta di una risposta ad una ambasceria che aveva sottoposto al parere dell'imperatore una gnome della boule e del demos, non un decreto.

20. Cfr. M.-F. Baslez, Les notables entre eux. Recherches sur les associations d'Athènes à l'époque romaine, in S. Follet (ed.), L'Hellénisme d'époque romaine, Parigi, 2004, p. 113; sui Paianistai cfr. J. H. Oliver, Paeanistae, in TAPhA, 71, 1940, p. 302-314; S.B. Aleshire, Asklepios at Athens, Amsterdam, 1991, p. 3340.

21. PIR ${ }^{2}$ C 821; Groag 1939, p. 133; Oliver 1973, p. 404; Guerber 1997, p. 218 (nr. 8)

22. IG, II-III' ${ }^{2}, 4215,1.3-5$.

23. Groag 1939, p. 137, parla di una estensione delle competenze del corrector alla supervisione finanziaria delle civitates liberae della provincia; il suo titolo in latino sarà stato legatus $A u$ gustorum pro praetore ad rationes putandas et corrigendum statum liberarum civitatium provinciae Achaiae. Secondo Guerber reiterato la curatela, con un breve intervallo di tempo tra i due incarichi.

Ti. Claudius Callippianus Italicus ${ }^{21}$ rappresenta un caso particolare. Di probabile origine grecoorientale, legatus Augustorum pro praetore di rango consolare, egli fu contemporaneamente curator et corrector delle città libere dell'Acaia nell'età di Settimio Severo e di Caracalla ${ }^{22}$. In questo caso la funzione di logistes si riferisce a tutte le città libe$\mathrm{re}^{23}$. È possibile, inoltre, se si accetta l'integrazione di FD III, 4255 proposta da C. Vatin, che Callippianus Italicus sia stato anche proconsole della provincia d'Acaia, e che abbia ricoperto le funzioni di corrector e curator delle città libere insieme al proconsolato $^{24}$. Egli fu anche governatore della provincia di Ponto e Bitinia tra il 202 e il 205 d.C. ${ }^{25}$. La posizione della curatela nella carriera consolare di Callippianus non è sicura; essa è stata infatti datata sia prima che dopo il governatorato della Bitinia $^{26}$.

Tra la fine del II e l'inizio del III sec. d.C. la polis di Trezene onorò M. Aurelius Olympiodorus, filosofo e logistes della città ${ }^{27}$. La dedica (IG, IV, 796) è degna di nota per il riferimento alla nomi-

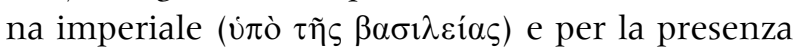
di una indicazione temporale relativa alla durata

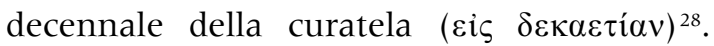

A Tegea sono attestati due logistai. M. Appale$n u s^{29}$, retore, onorato dalla città di Tegea $(I G, \mathrm{~V}, 2$ 155), è un rappresentante della famiglia degli $A p$ paleni, i quali appartenevano all'élite della colonia romana di Corinto; con ogni probabilità egli non era cittadino di Tegea e ricoprì la funzione di logi-

1997, p. 243, il fatto che alcuni correctores siano nello stesso tempo cuatores rei publicae indica che il corrector non aveva di per sè competenze finanziarie; quando era chiamato, in situazioni particolari, ad affrontare questioni finanziarie, allora assumeva in più il titolo di curator. Cfr. tuttavia B. E. Thomasson, Legatus, Stoccolma, 1991, p. 83, secondo il quale anche il corrector si occupava di questioni finanziarie; la differenza non stava nella sfera d'azione, bensì nelle modalità dell'azione : il curator esercitava una sorta di controllo, il corrector interveniva per apportare un miglioramento ( $V e r-$ besserung").

24. C. Vatin, Delphes à l'époque impériale (thèse inédite), 1968, p. 149-152 (cfr. Guerber 1997, p. 238).

25. $A E, 1939$, nr. 295

26. B. Rémy, Les fastes sénatoriaux des provinces romaines d'Anatolie au Haut-Empire, Parigi, 1988, p. 90 (nr. 80) e 91 (prima); Guerber 1997, p. 233, nr. 8 (dopo).

27. Roman Peloponnese I, ARG 49.

28. IG, IV, 796, l. 4-5; per la durata eccezionale vid. infra.

29. Roman Peloponnese I, ARC 8. 
stes "as an outside appointee $»^{30}$ tra la fine del II e l'inizio del III sec. d.C.

P. Aelius Leonides ${ }^{31}$, onorato anch'egli dalla boule e dal demos di Tegea (IG, V, 2 152), oltre alla funzione di logistes ricoprì quella di dekaprotos ${ }^{32}$. A differenza di Appalenus, egli era probabilmente un cittadino di Tegea ${ }^{33}$.

A Corone è attestato un logistes nella prima metà del III sec. d.C. (IG, V, 1 1398). C. Iulius Theagenes (II) ${ }^{34}$ era cittadino di Corone ${ }^{35}$ e apparteneva all'aristocrazia provinciale, come prova il fatto che fu generale della Lega achea ${ }^{36}$ (carica che ricoprì anche suo figlio).

A Cheronea, verso la metà del III sec. d.C., Cn. Curtius Dexippus pose una dedica alla madre, sacerdotessa (IG, VII, 3426) $)^{37}$; tra le cariche che accompagnano il suo nome è ricordata la funzione di

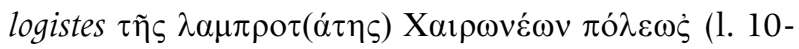
11). Con ogni probabilità Dexippus era cittadino di Cheronea, città nella quale tenne anche il sommosacerdozio imperiale a vita; fu inoltre beotarca per tre volte. Le cariche da lui ricoperte mostrano che egli apparteneva all'élite locale.

In una dedica proveniente da Sparta e databile alla prima metà del IV sec. d.C. (IG, V, $1524)^{38}$ è menzionato un logistes della colonia romana di Patrasso, Vitellius Valerinus ${ }^{39}$. Originario di Patrasso, Valerinus era un notabile locale

30. Spawforth 1974, p. 303. Spawforth ipotizza che il personaggio in questione sia il figlio, o più probabilmente il nipote, di Ti. Appalenus Anaxilaos, il quale fu hellanodikes ai giochi istmici per due volte negli anni ' 30 del II sec. d.C. Il gentilizio Appalenus è attestato solo in Apulia, a Barium, ed è probabilmente da ricondurre al milieu dei negotiatores dell'Oriente greco (cfr. A. J. S. Spawforth, Roman Corinth : the formation of a colonial elite, in A. D. Rizakis (ed.), Roman onomastics in the Greek East. Social and political aspects, Atene, 1996 (Meletemata, 21), p. 171 e nt. 24).

31. Roman Peloponnese I, ARC 2.

32. Questi funzionari corrispondono ai decemprimi delle province occidentali; cfr. S. Dmitriev, City government in Hellenistic and Roman Asia Minor, Oxford, 2005, p. 197-200 (cfr. anche G. P. Burton, The Imperial State and its impact on the role and status of local magitrates and councillors in the provinces of the empire, in L. de Blois (ed.), Administration, prosopography and appointment policies in the Roman Empire. Proceedings of the First Workshop of the international network impact of empire, Leiden June 28-July 1, 2000, Amsterdam, 2001, p. 207-209).

33. Cfr., a questo proposito, Spawforth 1974, p. 303, nt. $42:$ ( «It [scil. : l'iscrizione] illustrates the final stage in the evolution of the curatorship from a senatorial and equestrian office, as originally it had been, to a purely local one" (ma vid. infra, nt. 68).

34. Roman Peloponnese II, MES 228.

35. IvO, 451 (dedica in onore di C. Iulius Theagenes posta da suo che aveva compiuto il cursus municipale nella colonia e aveva raggiunto una posizione di primo piano nel consiglio cittadino, come si ricava dal titolo comes $^{40}$. Verosimilmente, proprio il prestigio raggiunto a livello locale da Valerinus gli permise di accedere all'ordine equestre, come sembra indicare il titolo kratistos, portato, a partire dal III e sempre di più nel IV sec. d.C., da cavalieri. Considerato che Valerinus è di Patrasso e che ha compiuto il cursus municipale nella colonia, si può affermare che ormai in questa fase la funzione di curator rei publicae si è trasformata in una magistratura locale (collocata al vertice della carriera municipale $)^{41}$.

Chiudiamo la nostra rassegna con tre logistai anonimi. Uno è attestato da una dedica onoraria proveniente da Andros e databile nel III sec. d.C. (IG, XII, 5 758). Un altro fu logistes di Sparta nel 359/60 d.C.; è menzionato in un rescritto del proconsole d'Acaia Publius Ampelius riguardante lavori di restauro nel teatro di Sparta, lavori per i quali fu creata una commissione apposita che riceveva i soldi dalle casse cittadine mediante il logistes (SEG, $\mathrm{XI}, 464)^{42}$. Infine, nel tardo IV sec. d.C., il proconsole d'Acaia Decimius Secundinus scrisse al curator et defensor Amfissensium (sic) una lettera (in latino) riguardante l'occupazione abusiva dell'aqua publica $(I L S, \quad 5794)^{43}$.

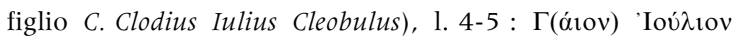

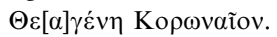

36. IvO, 451, 1. 6-7.

37. Cfr. J. M. Fossey, Some Imperial Statue Bases from the South West Kopais, in H. Kalcyk, B. Gullath e A. Graeber (a cura di), Studien zur Alten Geschichte (Festschrift Lauffer), I, Roma, 1986, p. 258, nr. 9.

38. A. D. Rizakis, Achaie II, Atene, 1998 (Meletemata, 25), p. 300, nr. $365^{*}$

39. Roman Peloponnese II, LAC 723. Sul gentilizio Vitellius cfr. RE, IX, A.1, 1961, c. 383-384; H. Solin e O. Salomies, Repertorium nominum gentilium et cognominum Latinorum, Hildesheim-Zurigo-New York, 1988, p. 211; è attestato a Corinto [Roman Peloponnese I, p. 399, nrr. 651-652 (II-III d.C.)].

40. Cfr. Cod. Theod., 12, 1, 127. Il titolo comes (civitatis) finirà per rimpiazzare l'espressione curator civitatis; cfr. RE, IV.1, 1900, c. 642 .

41. Cfr. Cod. Theod., 12, 1, 20 (331 d.C.) : tra i requisiti richiesti ai decurioni per diventare curator rei publicae c'è in primo luogo l'aver ricoperto tutti gli altri munera cittadini; cfr. Camodeca 1980, p. 481 (e nt. 120).

42. Cfr. D. Feissel e A. Philippidis-Braat, Inventaires en vue d'un recueil des inscriptions historiques de Byzance. III. Inscriptions $d u$ Péloponnèse, in TOMbyz, 9, 1985, p. 285-286, nr. 24; Roman Peloponnese II, LAC 657 [1].

43. Cfr. E. Groag, Die Reichsbeamten von Achaia in spätrömischer Zeit, Budapest, 1946, p. 56. 


\section{DISTRIBUZIONE CRONOLOGICA}

In un periodo di circa duecento anni (fine IIfine IV sec. d.C.), nella provincia d'Acaia sono attestati sedici curatores rei publicae; il primo (sicuramente datato) è attestato ad Orchomenos d'Arcadia nel 192/3 d.C., l'ultimo ad Amphissa verso la fine del IV sec. d.C. I sedici logistai non sono distribuiti uniformemente nell'arco dei due secoli. Se consideriamo solo quelli databili con una certa sicurezza (12 su 16), abbiamo la seguente situazione: sette curatores (nrr. $1 ; 2 ; 3 ; 4 ; 5 ; 6 ; 7$ ) furono attivi tra gli anni finali del II e quelli iniziali del III sec. d.C. (precisamente tra il 192/3 e il $212 / 3$ d.C.), due nella prima metà del III sec. d.C. (nrr. 11; 12), tre nel corso del IV sec. d.C. (nrr. 14; $15 ; 16)$. Per i restanti quattro la datazione è più incerta; comunque, due di essi possono essere collocati tra la fine del II e l'inizio del III sec. d.C. (nrr. 8; 9), gli altri due nel corso del III sec. d.C. (nrr. 10; 13). Da questo riepilogo della distribuzione cronologica dei logistai della provincia d'Acaia emerge un dato interessante : ben sette curatores o nove, se consideriamo anche M. Aurelius Olympiodorus e M. Appalenus (nrr. 8; 9) - su sedici si collocano in un breve lasso temporale, in pratica corrispondente al principato di Settimio Severo. Ci si può chiedere se questo dato sia indizio di una situazione storica particolare vissuta dalla provincia d'Acaia, in conseguenza della quale alcune città si trovarono ad affrontare problemi di natura amministrativo-finanziaria; due dei curatores databili tra fine II e inizio III d.C., ossia C. Licinius Telemachus e Ti. Claudius Callippianus Italicus, ricoprirono tra l'altro, contemporaneamente, la funzione di corrector delle città libere dell'Acaia, e la curatela assunta dal secondo sembra eccezionalmente ave- re riguardato tutte le città libere della provincia. Non mi sembra, invece, che sia lecito vedere in questa distribuzione cronologica dei logistai il risultato di qualche disposizione, riguardante i curatores rei publicae, ascrivibile a Settimio Severo ${ }^{44}$, o di una precisa politica imperiale, finalizzata ad esercitare, tramite i curatores, un maggiore controllo sull'Acaia (o in generale sulle province della pars orientale dell'Impero) ${ }^{45}$; in primo luogo, le fonti non attestano provvedimenti particolari in relazione ai curatores, o una politica di maggiore diffusione di questa funzione da parte di Settimio Severo; in secondo luogo, sarebbe sbagliato a priori ritenere i logistai attestati in Acaia una prova di una diminuzione dell'autonomia locale e di un più stretto controllo da parte del potere centrale ${ }^{46}$. Inoltre, tenuto conto dell'esiguità del campione statistico per l'Acaia (solo sedici curatores nell'arco di due secoli) e della componente di casualità dovuta alla natura fortuita dei rinvenimenti epigrafici ed insita inevitabilmente in qualsiasi analisi basata sul dato delle iscrizioni, la concentrazione di logistai che si registra nella provincia d'Acaia tra gli anni finali del II e quelli iniziali del III sec. d.C. potrebbe essere, almeno in parte, semplicemente frutto del $\mathrm{caso}^{47}$. Comunque, in quel periodo in Acaia diverse città conobbero, evidentemente, situazioni di squilibrio amministrativo-finanziario tali da determinare l'intervento di funzionari imperiali, compresi due correctores (legati imperiali di rango consolare o pretorio $)^{48}$. Colpisce, in particolare, il caso di Atene, che nel giro di pochi anni ebbe due curatores (nrr. 5; 6 - senza considerare Callippianus Italicus); per questa città, nel periodo in questione, disponiamo di alcuni indizi (la notizia dell'Historia Augusta ${ }^{49}$ secondo la quale Settimio Severo, che era stato offeso dagli Ateniesi,
44. Si può qui richiamare la notizia, riportata dall'Historia Augusta, secondo la quale Marco Aurelio nominò per varie comunità curatores di rango senatorio; Hist. Aug., Aur., 11, 2 : curatores multis civitatibus, quo latius senatorias tenderet dignitates, a senatu dedit.

45. In Asia Minore circa un quarto dei trentanove logistai censiti da Burton prima del 260 d.C. si datano nell'epoca di Settimio Severo e di Caracalla (Burton 1979, p. 482-483).

46. Negli ultimi anni diversi studi hanno rimesso in discussione "l'image couramment admise des curateurs fossoyeurs de l'autonomie municipale» (Jacques 1984, p. 5); cfr. ibid., p. XXX-XXXIV; 259 s. (part. 288-289 e 299-300); Camodeca 1980, p. 488-489; Eck 1999, p. 228. Le prerogative dei curatori e il carattere straordinario e transitorio dell'istituzione (almeno fino alla fine del III-inizio del IV sec. d.C.) non consentono di considerarli come affossatori dell'autonomia locale; cfr. Jacques - Scheid 1992, p. 339-340.

47. Comunque va notato, en passant, che, almeno in Occidente, i curatores rei publicae si concentrano soprattutto tra la fine dell'età antonina e l'epoca severiana (Jacques 1984, p. 4-5).

48. Cfr. Guerber 1997, p. 243, nt. 245 (in relazione a Ti. Claudius Callippianus Italicus, il quale, forse, congiuntamente alla correctura e alla curatela delle città libere dell'Acaia, ricoprì anche il proconsolato della stessa) : "Ce cumul de fonctions n'a pas pour source unique l'existence de problèmes financiers".

49. Hist. Aug., Sept. Sev., 3, 7. 
una volta salito al potere avrebbe tagliato i privilegia della città, e l'aumento del numero degli anni di "anarchia" nel tardo II e nel III sec. d.C. $)^{50}$ di una non facile situazione economica - del resto incominciata ben prima - la quale potrebbe aver determinato il presentarsi di problemi e il conseguente intervento di curatores.

Ad ogni modo, qualsiasi considerazione sulla maggiore diffusione dei curatores rei publicae nell'Impero in un preciso momento storico, e magari su iniziativa particolare di un princeps, imporrebbe una visione generale della distribuzione quantitativa di questi funzionari nelle province, in relazione alle linee-guida della politica adottata dai vari imperatori, il che esula dai limiti e dallo scopo del presente studio.

\section{ORIGINE GEOGRAFICA E CONDIZIONE SOCIALE}

Di norma, come è noto, i curatores rei publicae erano originari (e/o cittadini) di una città diversa da quella nella quale ricoprivano il loro incarico; questa situazione sembra essere la regola, non priva di eccezioni, fino ad un certo momento, quando quella di curator si trasformò in una magistratura municipale ricoperta dai membri dei locali consigli. Per quanto riguarda i logistai da noi considerati, solo in alcuni casi ci è nota la provenienza dei personaggi, mentre in altri casi si può cercare di inferire con un maggiore o minore grado di probabilità se il logistes in questione apparteneva alla comunità in cui rivestì la curatela; per esempio, nel caso di Cn. Curtius Dexippus, logistes di Cheronea verso la metà del III sec. d.C., il fatto che quella città abbia posto una dedica alla madre di Dexippus e in particolare che egli abbia rivestito il sommo-sacerdozio municipale rendono altamente probabile che fosse originario proprio di Cheronea. La provenienza dei logistai attestati in Acaia è genericamente riferibile all'ambito greco o greco-orientale; ciò considerato, la situazione si presenta come segue. In cinque casi origo del curator e sede dell'ufficio sono diverse (nrr. $4 ; 5 ; 6 ; 8$; $9)$, mentre in quattro casi queste coincidono (nrr. 10; 11; 12; 14). Per altri tre logistai (nrr. 1; 2; 3) la situazione è più incerta : potrebbero o meno

50. D. J. Geagan, Roman Athens: some aspects of life and culture I. 86 B.C.-A.D. 267, in ANRW, II, 7, 1, 1979, p. 406-407.

51. Flavius Saithidas (nr. 2) era sicuramente messenio, se non essere originari della città in cui furono curatores $^{51}$. Rimangono poi Ti. Claudius Callippianus Italicus (nr. 7), il quale fu curator di tutte le città libere dell'Acaia ed era forse di origine greco-orientale, e tre logistai anonimi (nrr. 13; 15; 16), due dei quali (nrr. 15; 16) si datano dopo la metà del IV sec. d.C., quando il curator rei publicae si è trasformato ormai in un funzionario locale. Ora, lasciando da parte Callippianus Italicus, i tre casi databili al IV secolo (nrr. 14; 15; 16) - quando la curatela di città è diventata una magistratura locale - e i casi incerti (nrr. $1 ; 2 ; 3 ; 13)$, abbiamo cinque logistai originari di una città diversa da quella della loro curatela (nrr. $4 ; 5 ; 6 ; 8 ; 9)$ e tre originari della stessa città (nrr. 10;11;12).

Quanto allo status sociale, i logistai attestati nella provincia d'Acaia sono tutti ${ }^{2}$ cittadini romani. Tre (nrr. $5 ; 6 ; 7)$ appartengono all'ordine senatorio (C. Licinius Telemachus e Ti. Claudius Subatianus Proculus, di rango pretorio; Ti. Claudius Callippianus Italicus, di rango consolare). Un secondo gruppo (nrr. $1 ; 2 ; 3 ; 4 ; 8 ; 9 ; 10 ; 11 ; 12$ ) è formato da nove logistai che appartengono all'aristocrazia municipale o provinciale. In effetti, almeno uno di questi, vale a dire $C$. Iulius Theagenes, logistes di Corone nella prima metà del III sec. d.C., può essere considerato un membro dell'élite socio-politica della provincia, dal momento che fu anche generale della Lega achea; del resto, va detto che esponenti delle aristocrazie municipali che furono logistai in città diverse dalla loro - come, ad esempio, il corinzio M. Appalenus o l'ateniese appartenente ai Claudii di Melite (nr. 4) - godevano evidentemente di un prestigio che oltrepassava i confini della loro patria.

Vitellius Valerinus (nr. 14) era un notabile appartenente all'élite di Patrasso; per quanto la sua condizione, in senso strettamente sociale, sia accostabile a quella dei personaggi del secondo gruppo, il fatto che egli abbia compiuto il cursus municipale nella colonia romana - ottenendo l'accesso all'ordine equestre - prima di diventarne curator mostra che in questa fase (prima metà del IV sec. d.C.) la curatela si era trasformata in una mera magistratura municipale. Infine, rimangono i tre logistai anonimi (nrr. 13; 15; 16), per i quali non si hanno informazioni.

proprio di Asine.

52. I tre logistai anonimi, ovviamente, sono esclusi dal computo. 
Ora, se escludiamo dal conto Vitellius Valerinus - che fu logistes quando la curatela di città non faceva più parte della carriera senatoria ${ }^{53}$ - e i logistai anonimi, vediamo che, su un totale di dodici curatores, tre sono senatori, nove notabili ${ }^{54}$. Notiamo quindi una prevalenza di notabili curatores rispetto a quelli di rango senatorio ${ }^{55}$. Non sembra lecito riscontrare un'evoluzione cronologica : le tre curatele senatorie si collocano in un breve lasso di tempo, mentre i curatores notabili si incontrano fin da subito. Piuttosto, va considerato che Callippianus Italicus fu curator di tutte le città libere dell'Acaia, che Licinius Telemachus e Subatianus Proculus furono, probabilmente non a caso, curatores di due tra le città più importanti della provincia, il primo ad Atene (forse per due volte), il secondo di nuovo ad Atene e a Patrasso ${ }^{56}$, che sia Callippianus che Telemachus, inoltre, erano legati imperiali i quali associarono la curatela alla carica di corrector - e la loro missione potrebbe inserirsi in un momento particolarmente delicato per la provincia e segnatamente per Atene ${ }^{57}$. Dunque, le tre curatele senatorie, concentrate tra l'altro in un breve lasso temporale, costituiscono, nel quadro delle curatele attestate in Acaia nel II e III sec. d.C., dei casi a parte. Tenuto conto di ciò, pur con tutta la cautela del caso dovuta all'esiguità e alla natura del campione epigrafico, sembra di poter riconoscere, nella scelta da parte dell'autorità imperiale dei curatores da assegnare alle città libere della provincia d'Acaia, una generale preferenza per esponenti delle aristocrazie municipali e dell'élite provinciale, anche della stessa città in cui avrebbero svolto la curatela (per almeno tre di essi origo e sede dell'ufficio coincidono $\left.{ }^{58}\right)$. Questi personaggi, in virtù della loro provenienza geografica e sociale, avevano verosimilmente più dimestichezza con le situazioni e le realtà locali, e l'autorità romana, rappresentata in provincia dal governatore, avrà preferito, almeno nella maggior parte dei casi, affidarsi ad essi per l'incarico di logistes. D'altronde, ciò non to-

53. Cfr. Camodeca 1980, p. 482.

54. Per tre di questi ultimi origo e sede dell'ufficio coincidono (nrr. 10;11;12), per altri tre sono diverse (nrr. $4 ; 8 ; 9)$, per i restanti tre l'origo è incerta (nrr. 1; 2; 3).

55. In Asia Minore, Burton 1979, p. 468, ha contato, fino al 260 d.C., sette curatori di rango senatorio e tre di rango equestre a fronte di undici curatori appartenenti all'élite politica e sociale della provincia.

56. In Italia la documentazione epigrafica mostra che in linea di massima le città più importanti erano affidate a curatores rei glie che, laddove l'importanza della città o la gravità della situazione lo richiedessero, potesse essere inviato un senatore (curatele di Proculus e di Telemachus ad Atene e a Patrasso, di Callippianus in tutte le città libere dell'Acaia).

Altre considerazioni (titolatura; durata della carica; cumulo di più curatele; funzioni dei logistai)

Il termine normalmente usato in Acaia per in-

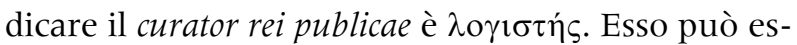
sere specificato dal sostantivo $\pi$ ó $\lambda 1 \varsigma$, usato da solo ( $I G$, V, 1 1398) o con l'etnico al genitivo plurale identificante la città (IG, II-III ${ }^{2}, 2963$; $I G$, VII, $3426)$; in un caso, al posto di $\pi$ ó $\lambda 1 \varsigma$, ricorre il sostantivo ко $\lambda \omega v i ́ \alpha(I G, \mathrm{~V}, 1524)^{59}$. In tre casi è usata

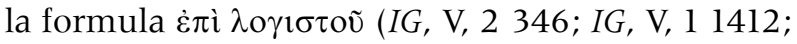
$I G$, XII, 91235 A). Talvolta, al posto del sostantivo logistes si trova il participio $(I G, \mathrm{~V}, 2$ 152, l. $3: \lambda \mathrm{o} \gamma \mathrm{l}-$

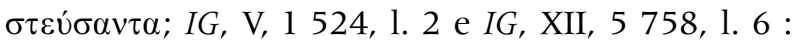

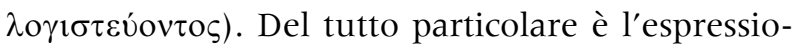
ne usata per indicare la curatela in una dedica proveniente da Trezene (IG, IV, 796) : $\tau \varepsilon \mu \eta \theta \varepsilon \dot{v} \tau \alpha \alpha \lambda_{\mathrm{o}-}$ $\gamma 1 \sigma \tau \varepsilon^{\prime} \alpha$; da notare che questo è anche l'unico caso in cui viene specificata la nomina imperiale (ن் $\tau \tilde{\eta} \varsigma \beta \alpha \sigma \imath \lambda \varepsilon i \alpha \varsigma)$. Ad Amphissa, in una iscrizione in latino (ILS, 5794), troviamo il termine curator seguito dall'etnico al genitivo plurale.

La durata della curatela viene specificata in un solo caso : nella dedica trezenia sopra citata, relativa al logistes M. Aurelius Olympiodorus, è infatti presente l'indicazione "per dieci anni" ( $\varepsilon i \varsigma \delta \varepsilon \kappa \alpha \varepsilon$ tíav). Normalmente i curatores rei publicae rimanevano in carica per periodi piuttosto brevi; verosimilmente, la loro missione durava lo stretto tempo necessario a raddrizzare la situazione di difficoltà. Se il loro incarico si protraeva per più tempo, bisogna immaginare che essi esercitassero solo un blando controllo sulla città; è questo probabilmente il caso di Olympiodorus ${ }^{60}$. Anche la curatela

publicae senatorii; cfr. Camodeca 1980, p. 479.

57. Forse il primo fu contemporaneamente anche proconsole d'Acaia.

58. Vid. supra, nt. 54.

59. In $I G, \mathrm{II}-\mathrm{III}^{2}, 1077$, l. 33, viene utilizzato, con lo stesso significato di $\pi$ ó $\lambda \iota \varsigma$, il sostantivo $\pi \alpha \tau \rho$ ṕs.

60. Cfr. Jacques 1984, p. 283 s. Secondo Pflaum 1960-1961, p. 523-531, nr. 193, M. Aurelius Mindius Matidianus Pollio sarebbe stato curator di Nicomedia, Prusa e Nicea per trent'anni; ma cfr. le obiezioni di Jacques 1984, p. 283, nt. 107. 
esercitata ad Atene da C. Licinius Telemachus potrebbe essersi prolungata per qualche anno; infatti, due iscrizioni databili rispettivamente al 209/10 e al 212/3 d.C. ${ }^{61}$ lo menzionano entrambe come curator. Tuttavia, in questo caso, nulla ci impedisce di pensare ad una iterazione della carica da parte di Telemachus nel giro di pochi anni.

Riguardo all'eventuale cumulo di più curatele, Subatianus Proculus, curator Atheniensium et Patrensium, potrebbe aver ricoperto congiuntamente le due curatele ${ }^{62}$; è improbabile invece che il logistes di Epidauro, Cheronea, Coronea e Tebe, attestato da un'iscrizione di Eleusi $\left(I G, \mathrm{II}-\mathrm{III}^{2}, 3610\right)$, abbia ricoperto quattro curatele contemporaneamente, tanto più che nell'iscrizione ricorre l'espressione

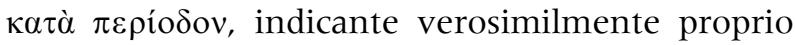
una successione temporale delle curatele ${ }^{63}$. Ti. Claudius Callippianus Italicus fu curator (e corrector) di tutte le città libere dell'Acaia (oltre che, forse, proconsole della stessa).

Infine, per quanto riguarda le funzioni dei logistai attestati nella provincia d'Acaia, dai documenti epigrafici - per lo più dediche onorarie che riportano il nome e il titolo del personaggio che ricopre la curatela - è possibile ricavare solo qualche scarno indizio. Ad Atene, nel 209/10 d.C., il curator C. Licinius Telemachus dovette fornire il nulla-osta alle spese legate alle celebrazioni del dies imperii di Geta, prima che il decreto ad esse relativo - formulato sotto forma di gnome dal consiglio dell'Areopago, dal consiglio dei 500 e dall'assemblea popolare - potesse diventare operativo. A Sparta, nel 359/60 d.C., il logistes gestiva i fondi cittadini necessari allo svolgimento di lavori nel teatro. Ad Amphissa, nel tardo IV sec. d.C., il curator et defensor Amfissensium (sic) intervenne, su indicazione del proconsole d'Acaia, contro l'occupazione abusiva di cisterne dell'acquedotto cittadino. Infine, è possibile che, nei tre casi di dediche imperiali in cui ricorre l'espressione $\dot{\varepsilon} \pi \grave{\imath} \lambda$ o $\gamma 1 \sigma \tau o \tilde{v}$, quest'ultimo abbia dato l'autorizzazione all'erezione su suolo pubblico della statua dell'imperatore. In sostanza, queste funzioni rientrano nelle competenze del $c u$ -

61. IG, II-III'2, 1077; IG, II-III' ${ }^{2}, 2963$

62. Secondo Camodeca 1980, p. 486-487, quando viene usata la congiunzione et e le città non sono più di due è molto probabile che si tratti di un cumulo di curatele.

63. Cfr., ex. g., IGUR, 246 A, 1. 20-21 : $\tau \tilde{\omega} v \gamma \rho \alpha \mu \mu \alpha] \tau \dot{\varepsilon} \omega v \tau \tilde{\omega} v \kappa \alpha-$

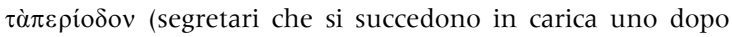
l'altro).

64. Come la costruzione di edifici, l'erezione di statue (su suolo rator rei publicae ricavabili dalle fonti epigrafiche, anche di altri contesti geografici, e da quelle giuridiche, e riguardanti in generale il controllo e la supervisione (temporanee) del patrimonio della comunità, quindi anche di tutte quelle attività che coinvolgevano i beni mobili e immobili della stes$\mathrm{sa}^{64}$.

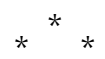

Se volessimo tentare, sulla base della esigua documentazione epigrafica a nostra disposizione, di fornire un identikit del curator rei publicae nelle città della provincia d'Acaia (prima del IV sec. d.C.), potremmo dire che egli è un esponente delle aristocrazie municipali e dell'élite socio-politica provinciale, il quale può essere cittadino della stessa città in cui ricopre la curatela. Su dodici logistai - escludendo i logistai del IV secolo e quelli anonimi - tre sono senatori, gli altri nove notabili appartenenti all'élite municipale o provinciale; di questi nove, tre sono estranei alla città in cui ricoprirono la curatela, altri tre sono della stessa città ${ }^{65}$. Nella prima metà del IV sec. d.C. si è ormai verificata una trasformazione nella natura del curator rei publicae, come mostra, in linea con le altre province dell'Impero, il caso di Vitellius Valerinus, il quale è un magistrato locale di Patrasso che ha svolto il cursus nella colonia romana prima di diventarne curator. Ora, è possibile, per la provincia d'Acaia, definire il momento in cui il curator rei publicae si trasformò da "funzionario" imperiale in un magistrato locale? Ritengo che la documentazione epigrafica - sia per il numero troppo esiguo delle attestazioni sia per la loro natura (assenza per alcuni dei logistai di informazioni precise su origine geografica, condizione sociale e cronologia) - non permetta di rispondere a questa domanda. Si potrebbe notare, sulla scorta della convinzione, a lungo prevalente, che riconosce nell'età di Severo Alessandro la fase di passaggio dal «vecchio" al "nuovo" curator rei publicae, che i tre logistai notabili, cittadini della stessa città dove eserci-

pubblico), l'organizzazione di concorsi, l'esecuzione di fondazioni evergetiche, ecc.; cfr. Camodeca 1980, p. 464 s.; Jacques 1984, p. 290-298; Jacques - Scheid 1992, p. 344; Eck 1999 , p. 212-227; cfr. anche E. Guerber e M. Sartre, Un logistès à Canatha (Syrie), in ZPE, 120, 1998, p. 97.

65. I restanti tre potrebbero essere, o non essere, originari della stessa città di cui furono curatores; vid. supra. 
tano la curatela (nrr. 10; 11; 12), si collocano nel corso del III sec. d.C. (e di questi almeno due entro la metà del secolo); ma basta questo per indicare la prima metà del III sec. d.C. come il momento della trasformazione del curator rei publicae in Acaia? Bisogna essere cauti; non è escluso che proprio i primi logistai attestati in Acaia (nrr. 1; 2; 3) - sui quali purtroppo non abbiamo informazioni sicure riguardo alla origine geografica - fossero cittadini della stessa città in cui esercitarono la curatela. Del resto, l'identità di origo del curator rei publicae e di sede dell'ufficio non è un elemento sufficiente a provare il cambiamento; in Occidente, ad esempio, troviamo già nell'età di Adriano curatores che ricoprirono la curatela nella loro città ${ }^{66}$. Infine, la communis opinio, che individua in generale nell'età di Severo Alessandro il momento della trasformazione del curator rei publicae, è stata messa in dubbio sia per le province occidentali che per l'Asia Minore ${ }^{67}$.

Piuttosto, dal punto di vista della caratterizzazione sociale e politica, escludendo i tre senatori - che tra l'altro, come ho cercato di dimostrare, rappresentano verosimilmente dei casi a parte - mi sembra che sia riscontrabile una sostanziale uniformità tra i logistai attestati in Acaia anteriormente al IV secolo d.C. (al di là del fatto che essi siano o meno cittadini della città in cui ricoprono la curatela); a ciò si aggiunga che le attestazioni epigrafiche ad essi relative non mostrano indizi della trasformazione della curatela in una semplice magistratura municipale (almeno nella prima metà del III sec. d.C.) ${ }^{68}$.

In conclusione, e sottolineando nuovamente la relatività delle considerazioni fin qui esposte, se da un lato l'esiguità del campione epigrafico e le incertezze cronologiche e interpretative che lo caratterizzano consentono solo di fornire spunti di carattere ipotetico, impedendo di definire con precisione il momento in cui si verificò un cambiamento nella figura del curator rei publicae in Grecia, dall'altro non mi sembra che i dati in nostro possesso autorizzino a collocare questo cambiamento nella prima metà del III sec. d.C.

Francesco CAMIA

\section{Abbreviazioni bibliografiche}

Burton 1979 = G. P. Burton, The Curator Rei Publicae : towards a reappraisal, in Chiron, 9, 1979, p. 465-488. Byrne 2003 = S. Byrne, Roman citizens of Athens, Lovanio, 2003.

Camodeca $1980=\mathrm{G}$. Camodeca, Ricerche sui curatores rei publicae, in $A N R W$, II, 13, 1980, p. 453-534.
Eck 1999 = W. Eck, L'Italia nell'Impero romano, Bari, 1999.

Groag 1939 = E. Groag, Die römischen Reichsbeamten von Achaia bis auf Diokletian, Vienna-Lipsia, 1939.

Guerber 1997 = E. Guerber, Les correctores dans la partie hellénophone de l'empire romain du règne de Trajan à

and equestrian office, as originally it had been, to a purely local one». Alla luce della presente indagine questa affermazione non mi sembra del tutto condivisibile, basata com'è, in pratica, solo sul confronto con il caso di M. Appalenus, logistes di Tegea tra la fine del II e l'inizio del III sec. d.C. e appartenente all'aristocrazia corinzia; comunque, la curatela di Leonides si data genericamente al III sec. d.C. All'epoca di Vitellius Valerinus, invece, la trasformazione del curator rei publicae è già sicuramente avvenuta; infatti, sebbene da un punto di vista strettamente sociale lo stesso Valerinus sia accostabile, in quanto membro dell'élite municipale, ai logistai del III secolo, tuttavia la sua curatela si caratterizza ormai come mera magistratura municipale. 
l'avènement de Dioclétien : étude prosopographique, in Anatolia Antiqua, 5, 1997, p. 211-248.

Jacques 1984 = F. Jacques, Le privilège de liberté. Politique impériale et autonomie municipale dans les cités de l'Occident romain (161-244), Roma, 1984 (Collection de l'École française de Rome, 76).

Jacques - Scheid 1992 = F. Jacques e J. Scheid, Roma e il suo Impero, Roma-Bari, 1992.

Mason 1974 = H. J. Mason, Greek terms for Roman institutions, Toronto, 1974.

Oliver 1973 = J. H. Oliver, Imperial commissioners in Achaia, in GRBS, 14, 1973, p. 389-405.
Pflaum 1960-1961 = H. G. Pflaum, Les carrières procuratoriennes équestres sous le Haut-Empire romain, Parigi, 1960-1961.

Roman Peloponnese I = A. D. Rizakis, S. Zoumbaki e M. Kantirea, Roman Peloponnese I. Roman personal names in their social context, Atene, 2001 (Meletemata, 31).

Roman Peloponnese II = A. D. Rizakis, S. Zoumbaki e Cl. Lepenioti, Roman Peloponnese II. Roman personal names in their social context, Atene, 2004 (Meletemata, 36).

Spawforth 1974 = A. J. S. Spawforth, The Appaleni of Corinth, in GRBS, 15, 1974, p. 295-303. 


\section{Appendice}

I CURATORES REI PUBLICAE ATTESTATI

NELLA PROVINCIA D'ACAIA

\begin{tabular}{|c|c|c|c|c|c|c|}
\hline $\mathrm{N}^{\circ}$ & Nome & Condizione sociale & Origine $^{1}$ & Città della curatela & Datazione $^{2}$ & Attestazioni \\
\hline 1 & $\begin{array}{l}\text { T. Flavius } \\
\text { Philargyrus }\end{array}$ & notabile & $?$ & $\begin{array}{l}\text { Orchomenos } \\
\text { (Arcadia) }\end{array}$ & 192/3 d.C. & $I G, \mathrm{~V}, 2346$ \\
\hline 2 & Flavius Saithidas & $\begin{array}{l}\text { notabile (élite } \\
\text { messenia) }\end{array}$ & $?$ & Asine & 193-195 d.C. & $I G, \mathrm{~V}, 11412$ \\
\hline 3 & Claudius Satyrus & notabile & $?$ & Hestiea (Eubea) & 195-198 d.C. & $I G, \mathrm{XII}, 91235 \mathrm{~A}$ \\
\hline 4 & $\begin{array}{l}\text { Ti. Claudius Sospis } \\
\text { o Ti. Claudius } \\
\text { Philippus }\end{array}$ & $\begin{array}{l}\text { notabile (élite } \\
\text { ateniese) }\end{array}$ & $\mathrm{X}$ & $\begin{array}{l}\text { Epidauro; } \\
\text { Cheronea; } \\
\text { Coronea; Tebe. }\end{array}$ & Fine II sec. d.C. & $I G, \mathrm{II}-\mathrm{III}^{2}, 3610$ \\
\hline 5 & $\begin{array}{l}\text { Ti. Claudius } \\
\text { Subatianus Proculus }\end{array}$ & $\begin{array}{l}\text { senatore (di rango } \\
\text { pretorio) }\end{array}$ & $X$ & Atene e Patrasso & 205/6 d.C. & $I L S, 9488$ \\
\hline 6 & $\begin{array}{l}\text { C. Licinius } \\
\text { Telemachus }^{4}\end{array}$ & $\begin{array}{l}\text { senatore (di rango } \\
\text { pretorio) }\end{array}$ & $\mathrm{X}$ & Atene & 209/10-212/3 d.C. & 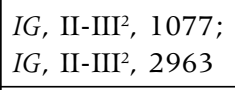 \\
\hline 7 & $\begin{array}{l}\text { Ti. Claudius } \\
\text { Callippianus } \\
\text { Italicus }^{5}\end{array}$ & $\begin{array}{l}\text { senatore (di rango } \\
\text { consolare) }\end{array}$ & & $\begin{array}{l}\text { Città libere } \\
\text { dell'Acaia }\end{array}$ & Intorno al 200 d.C. & $I G, \mathrm{II}-\mathrm{III}^{2}, 4215$ \\
\hline 8 & $\begin{array}{l}\text { M. Aurelius } \\
\text { Olympiodorus }\end{array}$ & notabile & $\mathrm{X}$ & Trezene & $\begin{array}{l}\text { Fine II-inizio } \\
\text { III sec. d.C. }\end{array}$ & $I G, \mathrm{IV}, 796$ \\
\hline 9 & M. Appalenus & $\begin{array}{l}\text { notabile (élite } \\
\text { corinzia) }\end{array}$ & $X$ & Tegea & $\begin{array}{l}\text { Fine II-inizio } \\
\text { III sec. d.C. }\end{array}$ & $I G, \mathrm{~V}, 2155$ \\
\hline 10 & P. Aelius Leonides & notabile & $\mathrm{O}$ & Tegea & III sec. d.C. & $I G, \mathrm{~V}, 2152$ \\
\hline 11 & C. Iulius Theagenes & $\begin{array}{l}\text { notabile } \\
\text { (aristocrazia } \\
\text { provinciale) }{ }^{6}\end{array}$ & $\mathrm{O}$ & Corone & $\begin{array}{l}\text { Prima metà } \\
\text { III sec. d.C. }\end{array}$ & $I G, \mathrm{~V}, 11398$ \\
\hline 12 & $\begin{array}{l}\text { Cn. Curtius } \\
\text { Dexippus }\end{array}$ & notabile & $\mathrm{O}$ & Cheronea & $\begin{array}{l}\text { Ca. metà } \\
\text { III sec. d.C. }\end{array}$ & $I G$, VII, 3246 \\
\hline 13 & $?$ & $?$ & $?$ & Andros & III sec. d.C. & $I G$, XII, 5578 \\
\hline 14 & Vitellius Valerinus & notabile (eques) & $\mathrm{O}$ & Patrasso & $\begin{array}{l}\text { Prima metà } \\
\text { IV sec. d.C. }\end{array}$ & $I G \mathrm{~V}, 1,524$ \\
\hline 15 & $?$ & $?$ & $?^{7}$ & Sparta & 359/60 d.C. & SEG, XI, 464 \\
\hline 16 & $?$ & $?$ & $?$ & Amphissa & $\begin{array}{l}\text { Seconda metà } \\
\text { IV sec. d.C. }\end{array}$ & ILS, 5794 \\
\hline
\end{tabular}

1. In questa colonna la "X» indica che origo del curator e sede dell'incarico sono diverse, mentre la « $\mathrm{O}$ » indica che origo e sede coincidono.

2. Un intervallo di più anni (es. : 193-195 d.C.) non va inteso necessariamente come la durata della curatela, bensì come il periodo in cui essa può essere collocata (talvolta ipoteticamente).

3. Sull'identità di questo logistes vid. supra, nt. 9.

4. Ricoprì, congiuntamente alla curatela, la funzione di corrector delle città libere dell'Acaia.

5. Ricoprì, congiuntamente alla curatela, la funzione di corrector delle città libere dell'Acaia e forse anche il proconsolato della stessa.

6. C. Iulius Theagenes è stato generale della Lega achea.

7. Questo personaggio ed il seguente saranno stati cittadini della stessa città in cui ricoprirono la curatela, dal momento che si datano nel IV secolo, quando la curatela di città si era ormai trasformata in una magistratura municipale. 